\title{
Sensor-Activity Relevance in Human Activity Recognition with Wearable Motion Sensors and Mutual Information Criterion
}

\author{
Oğuzcan Dobrucalı and Billur Barshan
}

\begin{abstract}
Selecting a suitable sensor configuration is an important aspect of recognizing human activities with wearable motion sensors. This problem encompasses selecting the number and type of the sensors, configuring them on the human body, and identifying the most informative sensor axes. In earlier work, researchers have used customized sensor configurations and compared their activity recognition rates with those of others. However, the results of these comparisons are dependent on the feature sets and the classifiers employed. In this study, we propose a novel approach that utilizes the time-domain distributions of the raw sensor measurements. We determine the most informative sensor types (among accelerometers, gyroscopes, and magnetometers), sensor locations (among torso, arms, and legs), and measurement axes (among three perpendicular coordinate axes at each sensor) based on the mutual information criterion.
\end{abstract}

\section{Introduction}

Automatic recognition of human activities has received considerable attention in the recent years. The ambiguous nature of daily human activities makes their correct recognition a challenging problem: Any specific activity can be performed in different styles by different people, a person can perform multiple activities simultaneously, and there is no one-to-one cause-effect relationship between consecutive activities [1]. Using a set of sensors and intelligent detection algorithms, activities can be automatically recognized and classified. In supervised approaches, activities are

O. Dobrucalı $(\varangle) \cdot$ B. Barshan

Department of Electrical and Electronics Engineering, Bilkent University,

06800Ankara, Bilkent, Turkey

e-mail: dobrucali@ee.bilkent.edu.tr

B. Barshan

e-mail: billur@ee.bilkent.edu.tr 
recognized based on priorly trained recognition models, whereas in unsupervised approaches, no prior models are assumed. The application areas of human activity recognition include identification systems that use the individual's physical and behavioural attributes (e.g., gait patterns), surveillance systems detecting unusual human activities, interactive systems responding according to the users' behaviour, synthesis of human activities in entertainment and robotics industries, medical treatment, and autonomous nursing of the elderly and disabled people [2, 3]. In addition to these, some recent studies are focused on localization of pedestrians and recognition of their activities at the same time [4].

Visual data recorded by video cameras [3], motion data acquired from wearable inertial and magnetic sensors [2], and acoustic data captured from microphones and vibration sensors [5] are the measurement types widely used in human activity recognition. In some studies, sensors are embedded in the environment, limiting the mobility area of the user, whereas in others, they are worn on the body to directly acquire motion data. In the latter, each sensor unit comprises a set of sensors that may include accelerometers, gyroscopes, magnetometers, inclinometers, goniometers, and tilt switches [2]. Motion data are analysed while being captured at either a single or multiple sensor locations. Commonly used sensor locations in recent studies are the head, ears, shoulders, wrists, torso, waist, thighs, calves, and ankles. Wearable sensors are preferable because they provide unbounded monitoring area and do not interfere with privacy. On the other hand, the user may forget to put them on or may feel distressed and uncomfortable while wearing them [6].

Sensor configuration is a fundamental issue in using wearable motion sensors that involves specifying (1) the number and type of sensors employed in each sensor unit, (2) the positions where the sensor units are placed, and (3) the axes along which each sensor provides informative measurements. To boost the overall recognition performance, feature sets in the time- and frequency-domains are commonly determined with respect to fixed sensor configurations [7]. The missing aspect here is an objective criterion for selecting a suitable sensor configuration. Various sensor configurations have been proposed in the literature. A couple of comparative studies [8-11] investigate sensor configurations and feature sets in terms of recognition accuracy. Optimal feature sets are selected while the sensor configuration is employed at full capacity $^{1}$ and vice versa. Consequently, any choice of sensor configuration seems to be highly dependent on the feature set. This fact, for instance, is explicitly shown in [7], where the authors compare the recognition performances of different feature sets computed from the measurements captured at several locations. Employing a basic classifier, they show that the contributions of the subsets of the sensor locations to the recognition performance vary, as does the feature set.

In this study, we identify the most informative sensor configuration by using the time-domain distributions of raw sensor data and the mutual information criterion. The approach presented here is independent of any feature set and classifier constraint, making it more objective and reliable than those mentioned above.

\footnotetext{
${ }^{1}$ The sensor configuration at full capacity implies the configuration employing all available sensor types, sensor locations, and measurement axes.
} 
The rest of this article is organized as follows: In the next section, we briefly describe the human activity dataset used in this study. In Sect. 3, we introduce the mutual information criterion for determining the informative sensor configurations. We provide the methodology and the results of the analysis in Sect. 4. Finally, we summarize the throughputs of this study and provide directions for future research in the last section.

\section{Human Activity Dataset}

The dataset analysed in this study is acquired by our research group at Bilkent University and used in some of our previous studies [8, 11, 12]. Descriptions of the 19 types of daily and sports activities included in this dataset and the physical characteristics of the eight subjects can be found in [8] and [12], respectively.

In this study, sensor units are placed at five locations on the body: torso (T), right arm (RA), left arm (LA), right leg (RL), and left leg (LL). Each sensor unit includes three tri-axial devices: an accelerometer (ACC), a gyroscope (GYRO), and a magnetometer (MAGN), whose measurement axes are aligned with a reference Cartesian coordinate frame, as depicted in Fig. 1a. The operating ranges of these devices are $\pm 18 g, \pm 1200^{\circ} / s$, and $\pm 75 \mu \mathrm{T}$, respectively [13]. Here, $g$ is the gravitational acceleration constant which is $9.80665 \mathrm{~m} / \mathrm{s}^{2}$. The positioning of the sensor units on the body is illustrated in Fig. 1b.

(a)

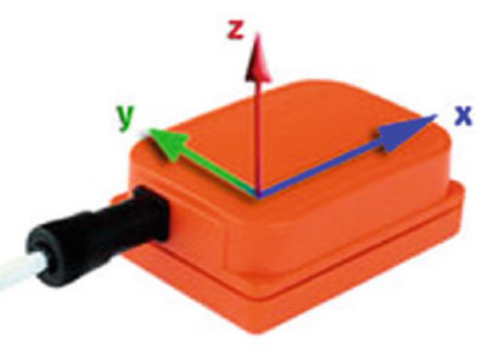

(b)

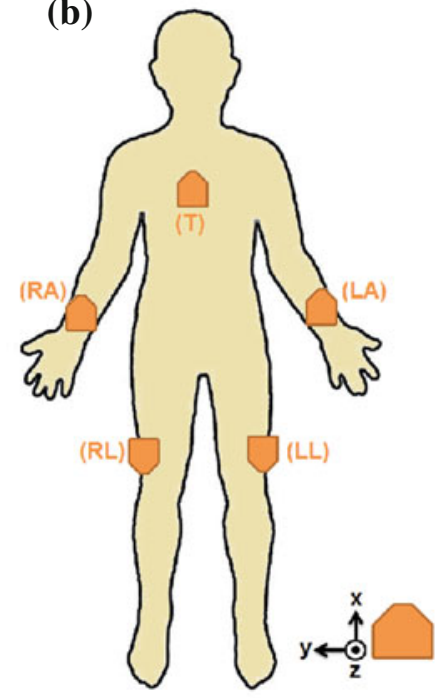

Fig. 1 a The sensor unit used in this study (reprinted from www.xsens.com/en/general/mtx) and b the unit positions on the subject's body. (The outline of the human body figure is taken from www. anatomyacts.co.uk/learning/primary/Montage.htm) 
The set of activities in the dataset is denoted by $\mathcal{A}$. When a subject performs an activity, the signals captured from the sensor axes on the subject's body are simultaneously recorded at $25 \mathrm{~Hz}$ sampling rate. Let each recorded discrete-time sequence be denoted by $U_{i j k c}[n]$ where $i \in\{1,2, \ldots, 5\}$ is the sensor location index representing $\{\mathrm{T}, \mathrm{RA}, \mathrm{LA}, \mathrm{RL}, \mathrm{LL}\}$ respectively, $j \in\{1,2,3\}$ is the sensor type index representing $\{\mathrm{ACC}, \mathrm{GYRO}, \mathrm{MAGN}\}$ respectively, $k \in\{x, y, z\}$ is the measurement axis symbol, $c \in \mathcal{A}$ is the activity symbol, and $n \in\{1,2, \ldots, 7500\}$ is the discrete-time index.

\section{Mutual Information}

Entropy is a measure of the information contained in a random variable in terms of its uncertainty. The entropy of a continuous random variable $\mathcal{X}$ is $H(\mathcal{X})=$ $-\int f_{\mathcal{X}}(x) \log _{2} f_{\mathcal{X}}(x) d x$ bits, where $f_{\mathcal{X}}$ is the marginal probability density function (PDF) of $\mathcal{X}$. Conditional entropy is a measure of the uncertainty of a random variable with the knowledge of another. The conditional entropy of $\mathcal{X}$, given another continuous random variable $\mathcal{Y}$ is $H(\mathcal{X} \mid \mathcal{Y})=-\iint f \mathcal{X}, \mathcal{Y}(x, y) \log _{2} f_{\mathcal{X}} \mid \mathcal{Y}(x \mid y) d x d y$ bits, where $f_{\mathcal{X}, \mathcal{Y}}$ and $f_{\mathcal{X} \mid \mathcal{Y}}$ are the joint and the conditional PDFs, respectively.

Based on entropy and conditional entropy, the mutual information between two random variables is the reduction of the uncertainty of one random variable using the knowledge about the other. Thus, the mutual information between $\mathcal{X}$ and $\mathcal{Y}$ is given by $\mathcal{I}(\mathcal{X}, \mathcal{Y})=\mathcal{H}(\mathcal{X})-\mathcal{H}(\mathcal{X} \mid \mathcal{Y})=\mathcal{H}(\mathcal{Y})-\mathcal{H}(\mathcal{Y} \mid \mathcal{X})$ bits. $\mathcal{I}(\mathcal{X}, \mathcal{Y})$ is bounded by zero and $\min \{H(\mathcal{X}), H(\mathcal{Y})\}$, therefore, it is a scalable measure. Mutual information is also the Kullback-Leibler distance between the joint PDF of the random variables and the product of their marginal PDFs. For continuous random variables $\mathcal{X}$ and $\mathcal{Y}$,

$$
\mathcal{I}(\mathcal{X}, \mathcal{Y})=\iint f_{\mathcal{X}, \mathcal{Y}}(x, y) \log _{2} \frac{f_{\mathcal{X}, \mathcal{Y}}(x, y)}{f_{\mathcal{X}}(x) f_{\mathcal{Y}}(y)} \mathrm{dx} \mathrm{dy}
$$

Consequently, mutual information becomes a measure of the dependence between two random variables, in terms of the information that one random variable provides about another [14]. Mutual information can be also used to measure the correlation between two random variables, instead of computing the linear correlation coefficient between them. The benefits of using mutual information are as follows [15]:

- Mutual information allows us to compute the correlation between multi-variate random vectors with either the same or different dimensions.

- Since mutual information depends on PDFs, it is not affected by the range of observed values of random variables.

- Mutual information can capture linear and non-linear dependencies between random variables. 


\section{Methodology}

To determine the most informative measurement axes, sensor types, and sensor locations for human activity recognition, we employ the mutual information criterion, which is suitable for computing the dependence between human activities and any combination of measurement types, including acceleration, rotational speed, and magnetic field strength.

We define the human activity classes as a discrete random variable, $\mathcal{C} \in \mathcal{A}$ where each activity in $\mathcal{A}$ is represented by a distinct symbol. $\mathcal{C}$ is uniformly distributed such that $\operatorname{Pr}\{\mathcal{C}=c\}=\frac{1}{|\mathcal{A}|}$ where $|\mathcal{A}|=19$. In the following subsections, we compute the mutual information between $\mathcal{C}$ and the measurements, grouped in different ways.

\subsection{Determining Informative Measurement Axes}

In this section, we compute the amount of activity-related information provided by each measurement axis of the sensors on the subject's body. For this purpose, we represent the output of each measurement axis with a distinct continuous random variable $\mathcal{M}_{i j k} \in \Re$. For each subject in the dataset, we define the mutual information between $\mathcal{C}$ and $\mathcal{M}_{i j k}$ according to Eq. (1) as

$$
\mathcal{I}_{l}\left(\mathcal{M}_{i j k}, \mathcal{C}\right)=\sum_{c \in \mathcal{A}} \int_{m_{i j k} \in \mathfrak{R}} f_{\mathcal{M}_{i j k}, \mathcal{C}}\left(m_{i j k}, c\right) \log _{2} \frac{f_{\mathcal{M}_{i j k}, \mathcal{C}}\left(m_{i j k}, c\right)}{f_{\mathcal{M}_{i j k}}\left(m_{i j k}\right) \operatorname{Pr}\{\mathcal{C}=c\}} d m_{i j k}
$$

where $l \in\{1,2, \ldots, 8\}$ is the subject index.

Since the different activity types are independent of each other, there is no dependence between the values of $\mathcal{C}$, and $f_{\mathcal{M}_{i j k}, \mathcal{C}}\left(m_{i j k}, c\right)=\left\{\bigcup f_{\mathcal{M}_{i j k} \mid \mathcal{C}}\left(m_{i j k} \mid c\right)\right.$ : $c \in \mathcal{A}$ \}. Here, $\hat{f}_{\mathcal{M}_{i j k} \mid \mathcal{C}}$, a histogram-based non-parametric estimate of $f_{\mathcal{M}_{i j k} \mid \mathcal{C}}$, is computed based on the corresponding observation sequence $U_{i j k c}[n]$. Then, $f_{\mathcal{M}_{i j k}}$ is estimated as $\hat{f}_{\mathcal{M}_{i j k}}\left(m_{i j k}\right)=\sum_{c \in \mathcal{A}} \hat{f}_{\mathcal{M}_{i j k} \mid \mathcal{C}}\left(m_{i j k} \mid c\right)$. Consequently, $\mathcal{I}_{l}\left(\mathcal{M}_{i j k}, \mathcal{C}\right)$ is approximated by a Riemann sum as follows:

$$
\mathcal{I}_{l}\left(\mathcal{M}_{i j k}, \mathcal{C}\right) \approx \sum_{c \in \mathcal{A}} \sum_{m_{i j k} \in M_{i j k}} \hat{f}_{\mathcal{M}_{i j k}, \mathcal{C}}\left(m_{i j k}, c\right) \log _{2} \frac{\hat{f}_{\mathcal{M}_{i j k}, \mathcal{C}}\left(m_{i j k}, c\right)}{\hat{f}_{\mathcal{M}_{i j k}}\left(m_{i j k}\right) \operatorname{Pr}\{\mathcal{C}=c\}} \delta m_{i j k}
$$

where $M_{i j k}$ is the set of histogram bin centre points and $\delta m_{i j k}$ is the sufficiently small histogram bin width over the range of $U_{i j k c}[n]$ for $c \in \mathcal{A}$.

For each measurement axis, the mean, the highest, and the lowest mutual information computed over all subjects are plotted in Fig. 2a. According to the figure, it 

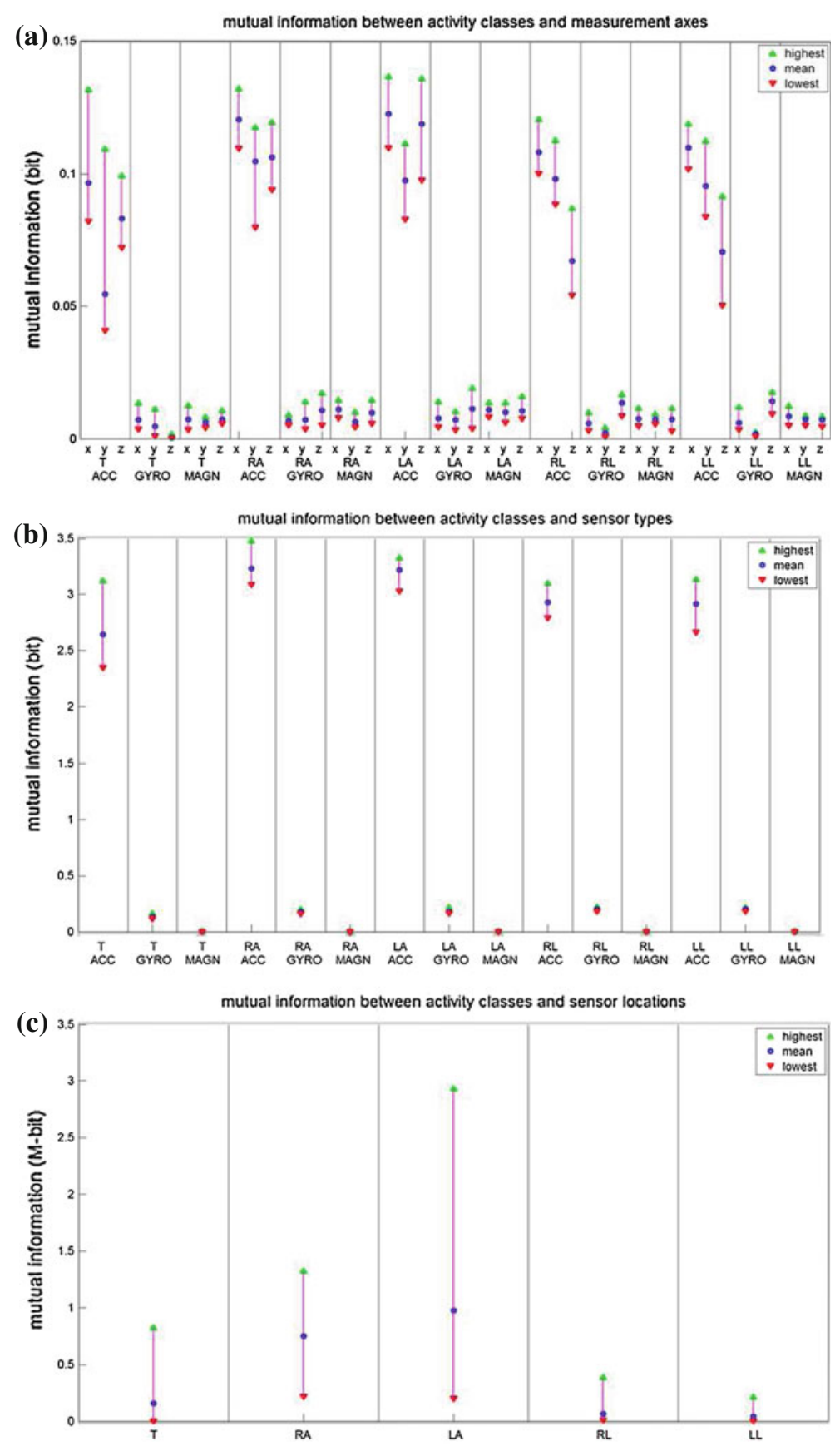

Fig. 2 The mean, the highest, and the lowest mutual information between the human activities and each a measurement axis, b sensor type, and $\mathbf{c}$ sensor location ( $T$ torso, $R A$ right arm, $L A$ left arm, $R L$ right leg, $L L$ left leg, $A C C$ accelerometer, GYRO gyroscope, MAGN magnetometer) 
is observed that at all sensor locations, the measurement axes of the accelerometers provide more activity-related information than those of gyroscopes and magnetometers. Furthermore, the $x$-axis of the accelerometers, which is perpendicular to the ground, is observed to contain higher activity-related information than the other two axes of the accelerometers, at all sensor locations.

\subsection{Determining Informative Sensor Types}

In this section, we compute the amount of activity-related information provided by each sensor type on the subject's body. Here, sensor type indicates the combination of the three measurement axes that belong to the same device (i.e., accelerometer, gyroscope, magnetometer) at the same sensor location. We represent each sensor type with a distinct continuous random vector $\mathcal{T}_{i j}=\left[\begin{array}{lll}\mathcal{M}_{i j x} & \mathcal{M}_{i j y} & \mathcal{M}_{i j z}\end{array}\right]^{\mathrm{T}} \in \mathfrak{R}^{3}$, where $\{x, y, z\}$ are the symbols of the measurement axes that belong to the sensor type $j$ at sensor location $i$. Based on Equation (1), the mutual information between $\mathcal{C}$ and $\mathcal{T}_{i j}$ for the $l$ th subject in the dataset is given by:

$$
\mathcal{I}_{l}\left(\mathcal{T}_{i j}, \mathcal{C}\right)=\sum_{c \in \mathcal{A}} \int_{t_{i j} \in \Re^{3}} f_{\mathcal{T}_{i j}, \mathcal{C}}\left(t_{i j}, c\right) \log _{2} \frac{f_{\mathcal{T}_{i j}, \mathcal{C}}\left(t_{i j}, c\right)}{f_{\mathcal{T}_{i j}}\left(t_{i j}\right) \operatorname{Pr}\{\mathcal{C}=c\}} \mathrm{dt}_{\mathrm{ij}}
$$

Since the different activities are independent of each other, $f_{\mathcal{T}_{i j}, \mathcal{C}}\left(t_{i j}, c\right)=$ $\left\{\bigcup f_{\mathcal{T}_{i j} \mid \mathcal{C}}\left(t_{i j} \mid c\right): c \in \mathcal{A}\right\}$. Here, $\hat{f}_{\mathcal{T}_{i j} \mid \mathcal{C}}$, a histogram-based non-parametric estimate of $f_{\mathcal{T}_{i j} \mid \mathcal{C}}$, is computed based on the corresponding observation sequences $U_{i j x c}[n]$, $U_{i j y c}[n]$, and $U_{i j z c}[n]$. Then, $f_{\mathcal{T}_{i j}}$ is estimated as $\hat{f}_{\mathcal{T}_{i j}}\left(t_{i j}\right)=\sum_{c \in \mathcal{A}} \hat{f}_{\mathcal{T}_{i j} \mid \mathcal{C}}\left(t_{i j} \mid c\right)$. Using these estimates, $\mathcal{I}_{l}\left(\mathcal{T}_{i j}, \mathcal{C}\right)$ is approximated by a Riemann sum as follows:

$$
\mathcal{I}_{l}\left(\mathcal{T}_{i j}, \mathcal{C}\right) \approx \sum_{c \in \mathcal{A}} \sum_{t_{i j} \in T_{i j}} \hat{f}_{\mathcal{T}_{i j}, \mathcal{C}}\left(t_{i j}, c\right) \log _{2} \frac{\hat{f}_{\mathcal{T}_{i j}, \mathcal{C}}\left(t_{i j}, c\right)}{\hat{f}_{\mathcal{T}_{i j}}\left(t_{i j}\right) \operatorname{Pr}\{\mathcal{C}=c\}} \Delta t_{i j}
$$

where $T_{i j}=\left(M_{i j x} \times M_{i j y} \times M_{i j z}\right)$ is the vector representing the set of histogram bin centres and $\Delta t_{i j}=\left(\delta m_{i j x} \cdot \delta m_{i j y} \cdot \delta m_{i j z}\right)$ is the histogram bin volume with sufficiently small histogram bin widths $\delta m_{i j x}, \delta m_{i j y}$, and $\delta m_{i j z}$ over the range of $U_{i j x c}[n], U_{i j y c}[n]$, and $U_{i j z c}[n]$ for $c \in \mathcal{A}$, respectively.

For each sensor type, the mean, the highest, and the lowest mutual information computed over all subjects are plotted in Fig. 2b. According to the figure, it is again observed that at all sensor locations, accelerometers provide considerably more activity-related information than gyroscopes and magnetometers. The information content of gyroscope measurements are slightly higher than those of magnetometers at all sensor locations. 


\subsection{Determining Informative Sensor Locations}

In this section, we compute the amount of activity-related information provided by each of the five sensor locations on the subject's body. Here, sensor location indicates the combination of the three sensor types at the same location. We represent each

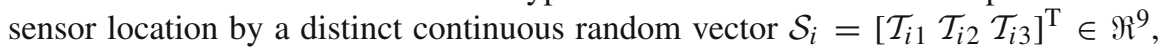
where $\{1,2,3\}$ are the indices of the sensor types at location $i$. For the $l$ th subject in the dataset, the mutual information between $\mathcal{C}$ and $\mathcal{S}_{i}$ is given by:

$$
\mathcal{I}_{l}\left(\mathcal{S}_{i}, \mathcal{C}\right)=\sum_{c \in \mathcal{A}} \int_{s_{i} \in \mathfrak{R}^{9}} f_{\mathcal{S}_{i}, \mathcal{C}}\left(s_{i}, c\right) \log _{2} \frac{f_{\mathcal{S}_{i}, \mathcal{C}}\left(s_{i}, c\right)}{f_{\mathcal{S}_{i}}\left(s_{i}\right) \operatorname{Pr}\{\mathcal{C}=c\}} \mathrm{d} s_{i}
$$

Because of the independence between the activities, $f_{\mathcal{S}_{i}, \mathcal{C}}\left(s_{i}, c\right)=\left\{\bigcup f_{\mathcal{S}_{i} \mid \mathcal{C}}\right.$ $\left.\left(s_{i} \mid c\right): c \in \mathcal{A}\right\}$. The $\hat{f}_{\mathcal{S}_{i} \mid \mathcal{C}}$ is a histogram-based non-parametric estimate of $f_{\mathcal{S}_{i} \mid \mathcal{C}}$ and computed based on the corresponding observation sequences $U_{i 1 x c}[n], U_{i 1 y_{c}}[n]$, $\ldots, U_{i 3 z c}[n]$. Then, the estimate of $f_{\mathcal{S}_{i}}$ is given by $\hat{f}_{\mathcal{S}_{i}}\left(s_{i}\right)=\sum_{c \in \mathcal{A}} \hat{f}_{\mathcal{S}_{i} \mid \mathcal{C}}\left(s_{i} \mid c\right)$ and $\mathcal{I}_{l}\left(\mathcal{S}_{i}, \mathcal{C}\right)$ is approximated by a Riemann sum as follows:

$$
\mathcal{I}_{l}\left(\mathcal{S}_{i}, \mathcal{C}\right) \approx \sum_{c \in \mathcal{A}} \sum_{s_{i} \in S_{i}} \hat{f}_{\mathcal{S}_{i}, \mathcal{C}}\left(s_{i}, c\right) \log _{2} \frac{\hat{f}_{\mathcal{S}_{i}, \mathcal{C}}\left(s_{i}, c\right)}{\hat{f}_{\mathcal{S}_{i}}\left(s_{i}\right) \operatorname{Pr}\{\mathcal{C}=c\}} \Delta s_{i}
$$

where $S_{i}=\left(T_{i 1} \times T_{i 2} \times T_{i 3}\right)$ is the vector representing the set of histogram bin centres and $\Delta s_{i}=\left(\Delta t_{i 1} \cdot \Delta t_{i 2} \cdot \Delta t_{i 3}\right)$ is the histogram bin volume. Because of memory allocation problems that arise when an enormous number of very small histogram bins are handled in $\mathfrak{R}^{9}$, the histogram bins in the estimation of $f_{\mathcal{S}_{i} \mid \mathcal{C}}$ cannot be set as small as in the estimations of $f_{\mathcal{M}_{i j k} \mid \mathcal{C}}$ and $f_{\mathcal{T}_{i j} \mid \mathcal{C}}$. Therefore, the approximation in Eq. (7) is not as accurate as in Eqs. (3) and (5). Despite this, the results are admissible for the comparison between the sensor locations.

For each sensor location, the mean, the highest, and the lowest mutual information computed over all subjects are plotted in Fig. 2c. According to the figure, it is observed that the arms provide the highest activity-related information, whereas the legs provide the lowest.

\section{Conclusion}

In this study, we employ a human activity dataset comprised of inertial and magnetic sensor measurements of 19 types of daily and sports activities and investigate the useful sensor types, sensor locations, and measurement axes of body-worn devices that provide high activity-related motion information.

In many human activity recognition studies, the measurements acquired from all sensors in the configuration are considered with equal significance. However, 
the results of this study indicate that the sensors do not contribute equal amount of information during the activity recognition process. The results of the proposed analyses can be used to determine the level of significance of each sensor.

Based on the mutual information criterion, we identify the linear acceleration measurements at all sensor locations as the most informative measurement type. Among the linear acceleration measurements, the measurement axes along the direction perpendicular to the ground are more informative than the others. In terms of sensor location, we identify the arms and the torso as the first and the second most informative locations, respectively. The legs are less informative compared to the extremities. The mutual information based approach proposed in this study can be used in selecting the most suitable sensor configuration among a set of possibilities.

Future cognitive systems are expected to be able to adapt to recent states, make estimates, and optimize their operating conditions autonomously. In parallel with this forecast, we envision a system which employs body-worn sensors that will be able to activate the informative sensors and suspend the others while simultaneously making activity decisions. In this way, the inputs to the recognition unit will be simplified to improve its decision accuracy. This study constitutes the first step of our research on such adaptive human activity recognition systems. In future work, our aim is to complete the relevance and redundancy relationships between sensor-feature-activity class trio.

\section{References}

1. Kim E, Helal S, Cook DJ (2010) Human activity recognition and pattern discovery. IEEE Pervas Comput (1):48-53

2. Preece SJ, Goulermas JY, Kenney LPJ, Howard D, Meijer K, Crompton R (2009) Activity identification using body-mounted sensors-a review of classification techniques. Physiol Meas 30(4):R1-R33

3. Turaga PK, Chellappa R, Subrahmanian VS, Udrea O (2008) Machine recognition of human activites: a survey. IEEE T Circ Syst Vid 18(11):1473-1488

4. Altun K, Barshan B (2012) Pedestrian dead reckoning employing simultaneous activity recognition cues. Meas Sci Technol 23(2):025103

5. Töreyin BU, Dedeoğlu Y, Çetin AE (2005) HMM based falling person detection using both audio and video. In: Sebe N, Lew M, Huang T (eds) Computer Vision in Human-Computer Interaction. Lecturer Notes Computational Science, Springer, Berlin, pp 211-220

6. Ganyo M, Dunn M, Hope T (2011) Ethical issues in the use of fall detectors. Ageing Soc 31(8):1350-1367

7. Preece SJ, Goulermas JY, Kenney LPJ, Howard D (2009) A comparison of feature extraction methods for the classification of dynamic activities from accelerometer data. IEEE T Bio-med Eng 56(3):871-879

8. Altun K, Barshan B (2010) Human activity recognition using inertial/magnetic sensor units. In: Salah A, Gevers T, Sebe N, Vinciarelli A (eds) Human Behavior Understanding. Lecture Notes Computational Science, Springer, Berlin, pp 38-51

9. Atallah L, Lo B, King RC, Gitang G-Z (2011) Sensor positioning for activity recognition using wearable accelerometers. IEEE T Bio-med Circ Syst 5(4):320-329 
10. Fish B, Khan A, Chehade NH, Chien C, Pottie G (2012) Feature selection based on mutual information for human activity recognition. 2012 IEEE International Conference Acoustics Speech. pp 1729-1732

11. Yüksek MC (2011) A comparative study on human activity classification with miniature inertial and magnetic sensors, MSc. thesis, Department of Electrical and Electronics Engineering, Bilkent University, Ankara, Turkey

12. Altun K, Barshan B, Tunçel O (2010) Comparative study on classifying human activities with miniature inertial and magnetic sensors. Pattern Recogn 43(10):3605-3620

13. Xsens Technologies BV (2010) MTi and MTx user manual and technical documentation, Document MT0100P, Revision O

14. Cover TM, Thomas JA (1991) Elements of Information Theory. Wiley, New York

15. Li W (1990) Mutual information functions versus correlation functions. J Stat Phys 60(5-6):823-837 